\title{
Analgesia pós-operatória com metadona em gatos: administração epidural e intramuscular
}

\author{
[Postoperative analgesia of methadone in cats: epidural and \\ intramuscular administration] \\ C.A. Bernardi, R.N. Cassu*, J.A.O. Balan, D.A. Costa, D. Fini \\ Faculdade de Ciências Agrárias - Universidade do Oeste Paulista - Presidente Prudente, SP
}

\begin{abstract}
RESUMO
Compararam-se os efeitos analgésico, sedativo e neuroendócrino decorrentes da administração epidural e intramuscular da metadona em gatas submetidas à ovariossalpingoisterectomia (OSH). Todos os animais foram tranquilizados com acepromazina, $0,1 \mathrm{mg} \mathrm{kg}^{-1} \mathrm{IM}$, seguindo-se a indução e manutenção anestésica com tiopental sódico, $12 \mathrm{mg} \mathrm{kg}^{-1} \mathrm{IV}$, e halotano, respectivamente. Após a estabilização anestésica, os animais foram distribuídos em três grupos, com oito animais cada, tratados com metadona pela via epidural (EP) ou intramuscular (IM) $0,2 \mathrm{mg} \mathrm{kg}^{-1}$, respectivamente, ou com solução salina (S) $0,2 \mathrm{~mL} \mathrm{~kg}^{-1}$. Foram avaliados: graus de analgesia e de sedação, necessidade de analgesia de resgate, efeitos adversos e concentração sérica de cortisol. Os graus de analgesia e de sedação e o cortisol não diferiram entre os tratamentos. Analgesia de resgate foi administrada quatro, nove e 11 vezes nos tratamentos EP, IM e S, respectivamente. Nos tratamentos IM e $S$, a suplementação analgésica foi necessária a partir da primeira hora pós-cirúrgica, enquanto no tratamento EP, somente a partir da terceira hora após a OSH. Conclui-se que a administração de metadona pela via epidural resulta em menor quantidade do analgésico pósoperatório, e que a analgesia é mais prolongada quando comparada à obtida pela via intramuscular, em gatas submetidas à $\mathrm{OSH}$.
\end{abstract}

Palavras-chave: gato, opioide, analgesia, metadona, extradural

\begin{abstract}
The aim of this study was to investigate the analgesic, sedative and neuroendocrine effects of epidural (EP) and intramuscular (IM) methadone in cats submitted to ovariohysterectomy. The pre-anesthetic medication was acepromazine $\left(0.1 \mathrm{mg} \mathrm{kg}^{-1}\right.$, IM) followed by induction of anesthesia with intravenous thiopental, $12 \mathrm{mg} \mathrm{kg}^{-1}$, and anesthesia maintenance with halothane. After anesthesia stabilization the cats were randomly assigned to three groups of eight animals each and received EP or IM methadone, 0.2mg


measurements, degree of sedation, rescue analgesia requirements, adverse effects and serum cortisol concentration were recorded. Cortisol, pain and sedation scores did not differ among the groups. Rescue analgesia was administered 4, 9 and 11 times in the EP, IM and S treatment, respectively. In the IM and S rescue analgesia treatments were required early (first hour postoperative), whereas in the EP treatment, additional analgesics were required after the third hour postoperative. In conclusion, methadone epidural reduced the postoperative analgesic requirements and produced longer analgesia when compared to intramuscular administration in cats undergoing ovariohysterectomy.
\end{abstract}

Keywords: cat, opioid, analgesia, methadone, extradural

Recebido em 26 de outubro de 2011

Aceito em 24 de novembro de 2011

* Autor para correspondência (corresponding author)

E-mail:renavarro@uol.com.br 


\section{INTRODUÇÃO}

A ovariossalpingoisterectomia (OSH) eletiva é uma das cirurgias de maior incidência em pequenos animais. A dor decorrente desse procedimento já foi demonstrada em diversos estudos (Rohrer Bley et al., 2004; Gassel et al., 2005; Brondani et al., 2009), porém ainda existe negligência com relação ao tratamento antálgico, sobretudo em felinos (Steagall et al., 2009).

Pesquisas desenvolvidas na década de 90 relataram a baixa frequência do tratamento da dor pós-operatória em felinos (Watson et al., 1996; Capner et al., 1999). Nos últimos anos, o emprego de analgésicos para a espécie felina aumentou de 13\% (2000) para 56\% (2005), conforme relatado em estudos retrospectivos (Joubert, 2006; Hewson et al., 2006). No entanto, apesar do incremento dispensado para o tratamento antálgico, muitos animais ainda recebem tratamento inadequado da dor pósoperatória, sobretudo em função do receio frente aos possíveis efeitos colaterais e informações limitadas a respeito da modulação nociceptiva para a espécie felina (Joubert, 2006). Ressalta-se, ainda, a preocupação com relação ao uso de opioides em gatos, em razão da possível excitação e euforia dose-dependente desencadeada por esses analgésicos, no entanto, quando doses adequadas são empregadas, esse efeito não é observado (Capner et al., 1999).

No intuito de buscar melhor esclarecimento em relação ao uso de analgésicos em gatos, muitos estudos têm sido desenvolvidos, revelando resultados promissores com o uso de opioides para o alívio da dor pós-operatória nessa espécie (Rohrer Bley et al., 2004; Gassel et al., 2005; Manfrinate et al., 2009; Steagall et al., 2009).

A metadona é um opioide agonista total, pertencente à classe difenilpropilamina, cujas propriedades farmacológicas são semelhantes às da morfina. No homem, a administração parenteral ou oral da metadona proporciona efeito analgésico de duração variável, oscilando entre quatro-seis horas até oito-20 horas, quando empregada em doses elevadas ou repetidas (Gourlay et al., 1986). Em cães e gatos o efeito analgésico da metadona após administração parenteral varia entre uma-quatro horas e 1,5-6,5 horas, respectivamente (Rohrer Bley et al., 2004; Leibetseder et al., 2006; Manfrinate et al., 2009;
Ferreira, 2010). Em razão da alta lipossolubilidade da metadona, o período de latência e a duração do efeito analgésico sofrem pouca alteração em função da administração epidural (Cousins e Mather, 1984). Em cães, o período de latência foi de 20 minutos, com duração do efeito analgésico por cerca de seis horas, após o uso epidural da metadona (Leibetseder et al., 2006). No entanto, há escassez na literatura sobre o uso epidural desse fármaco, para o alívio da dor pós-cirúrgica na espécie felina.

O objetivo deste estudo foi avaliar os efeitos analgésico, sedativo e neuroendócrino da metadona, quando utilizada pela via epidural comparativamente à via intramuscular em gatas submetidas à ovariossalpingoisterectomia eletiva (OSH).

\section{MATERIAL E MÉTODOS}

Após aprovação do Comitê de Ética e Pesquisa da instituição de origem (protocolo $\mathrm{n}^{\circ}$ 163/06) foram avaliadas 24 gatas, adultas, SRD, com peso médio de $2,8 \pm 2 \mathrm{~kg}$, clinicamente saudáveis, provenientes da rotina cirúrgica, encaminhadas para realização de ovariossalpingoisterectomia $(\mathrm{OSH})$ eletiva. Os animais foram selecionados para a pesquisa, mediante a normalidade do exame físico e testes laboratoriais (hemograma, ureia e creatinina).

Após período de jejum sólido e hídrico de 12 e três horas, respectivamente, todos os animais foram tranquilizados pela via intramuscular (IM) com $0,1 \mathrm{mg} \mathrm{kg}^{-1}$ de maleato de acepromazina (Acepran 0,2\%, Univet, São Paulo, Brasil). Trinta minutos após, foi realizada cateterização (Insyte, Becton Dickinson, Juiz de Fora, Brasil) da veia cefálica para indução anestésica e fluidoterapia com Ringer lactato (Solução de Ringer Lactato, HalexIstar, Goiânia, Brasil), em velocidade de $10 \mathrm{~mL} \mathrm{~kg}^{-1} \mathrm{~h}^{-1}$, mantida durante todo o procedimento cirúrgico. A indução anestésica foi realizada com $12 \mathrm{mg} \mathrm{kg}^{-1} \mathrm{de}$ tiopental sódico (Tiopentax, Cristália, Itapira, Brasil), seguindo-se a intubação orotraqueal (IO), com introdução de sonda sem balonete (número 3,5 - 4,0), selecionada em função do tamanho do animal, após instilação prévia de lidocaína spray (Xylestesin spray 10\% Cristália, Itapira, Brasil) para dessensibilização da região laríngea. Seguindo-se a IO, os animais 
foram submetidos à anestesia geral inalatória (Samurai 3300, Takaoka, São Paulo, Brasil), com halotano (Halotano, Cristália, Itapira, Brasil), sendo mantidos em ventilação espontânea, em circuito semiaberto (Baraca, Takaoka, São Paulo, Brasil), com fluxo de oxigênio de $400 \mathrm{~mL} \mathrm{~kg}^{-1}$ $\min ^{-1}$.

Após a estabilização da anestesia geral, os animais foram distribuídos ao acaso, em estudo cego, a um dos três tratamentos, com oito animais em cada: EP - metadona (Metadon, Cristália, Itapira, Brasil) na dose de $0,2 \mathrm{mg} \mathrm{kg}^{-1}$, diluída em solução salina a fim de perfazer o volume final de $0,2 \mathrm{~mL} \mathrm{~kg}^{-1}$, administrada pela via epidural; IM - metadona na dose de $0,2 \mathrm{mg}$ $\mathrm{kg}^{-1}$, diluída em solução salina a fim de perfazer um volume final de $0,2 \mathrm{~mL} \mathrm{~kg}^{-1}$, administrada pela via intramuscular; $S$ - solução salina na dose de $0,2 \mathrm{~mL} \mathrm{~kg}^{-1}$, administrada pelas vias intramuscular e epidural. Nos tratamentos EP e IM, foi administrada solução salina no volume de $0,2 \mathrm{~mL} \mathrm{~kg}^{-1}$ pelas vias intramuscular e epidural, respectivamente.

Os animais foram posicionados em decúbito esternal, estendendo-se os membros pélvicos cranialmente para punção do espaço epidural lombossacro (L7-S1), cuja localização foi confirmada pela ausência de resistência ao teste feito pela injeção de ar.

O efeito analgésico foi avaliado durante as primeiras 12 horas - às uma, três, seis e 12 horas - e após o término da cirurgia, mediante escala analógica visual (VAS) e escala analógica visual interativa (IVAS), utilizando-se uma linha de $100 \mathrm{~mm}$, onde o extremo esquerdo representa o animal sem sinais de dor e o extremo direito o máximo de dor (Rohrer Bley et al., 2004; Gassel et al., 2005; Brondani et al., 2009). Os escores atribuídos em função da VAS foram embasados no comportamento do animal dentro da gaiola, de modo que o animal com aspecto relaxado ou que se mantivesse próximo da porta da gaiola, na tentativa de interagir com o observador, recebeu escore 0 , enquanto os animais que se mantinham deitados, imóveis ou inconscientes após o período da recuperação pós-anestésica receberam escore 100. O uso da IVAS foi feito por meio da interação do animal com o pesquisador, de modo que, além da observação comportamental, a dor também foi avaliada em função da resposta à manipulação da ferida cirúrgica. $\mathrm{O}$ animal que não apresentou objeção à palpação da área afetada, com comportamento calmo e postura normal durante a manipulação, recebeu escore 0 , enquanto a ocorrência de vocalização intensa, com tentativa de morder o pesquisador no ato da palpação da área afetada, além da interação com comportamento agressivo durante a manipulação, foi considerada com escore 100 . Ademais, durante a palpação da ferida cirúrgica, a tensão da musculatura abdominal também foi avaliada, sendo que animais com tônus aumentado e rigidez eram considerados portadores de dor (Rohrer Bley et al., 2004).

Todos os animais, com VAS maior ou igual a 30 e/ou com IVAS maior ou igual a 50, receberam analgesia de resgate com metadona na dose de $0,2 \mathrm{mg} \mathrm{kg}^{-1}$ (IM). O número total, bem como o intervalo entre as administrações adicionais de metadona, foi registrado.

Foi avaliado também o grau de sedação no período pós-operatório, utilizando-se sistema de escore, em que 0 indica animal alerta e capaz de caminhar; 1, animal alerta, porém atáxico, incapaz de caminhar; 2, animal sonolento, porém com episódios de agitação; 3, animal muito sonolento (Rohrer Bley et al., 2004). Essa característica foi avaliada nos mesmos momentos usados para a avaliação do efeito analgésico.

A concentração sérica de cortisol foi mensurada por radioimunoensaio de fase sólida (Coat-ACount Cortisol - DPC), mediante colheita de sangue da veia jugular, que foi cateterizada, durante o procedimento anestésico, ao término da cirurgia de OSH, sendo protegida com bandagem, durante o período de avaliação. As colheitas foram realizadas, antes da medicação pré-anestésica, seis e 12 horas após o término da cirurgia.

Foi registrada a ocorrência de efeitos adversos como: sialorreia, vômito, midríase, hipertermia e euforia, durante o período de avaliação pósoperatória.

Após o período de observação de 12 horas, todos os animais foram tratados com meloxicam $\left(0,1 \mathrm{mg} \mathrm{kg}^{-1}\right.$, via $\left.\mathrm{SC}\right)$, com prescrição desse medicamento e de tramadol $\left(2 \mathrm{mg} \mathrm{kg}^{-1}\right.$, via SC), para uso em casa, durante três dias. Paralelamente, todos os animais foram tratados 
com enrofloxacina $\left(5 \mathrm{mg} \mathrm{\textrm {kg } ^ { - 1 }}\right.$, SC, a cada 12 horas), durante sete dias.

Foi utilizada análise de variância para comparação entre os grupos, com contrastes verificados pelo teste de Tukey. Para comparar os momentos dentro de cada grupo, foi utilizada análise de variância para amostras relacionadas, com contrastes verificados pelo teste de Tukey. Adotou-se a significância de 5\% (Zar, 1996).

\section{RESULTADOS}

Os três tratamentos foram comparáveis em relação aos dados demográficos e variáveis clínicas. Não houve diferença entre os tratamentos em relação ao peso, idade, tempo cirúrgico, tempo de extubação e período de recuperação pós-anestésica (Tab. 1).

Com relação aos escores de dor e de sedação, não houve diferença significativa entre os tratamentos e nem ao longo do tempo avaliado
(Tab. 2). Foram realizados quatro resgates de metadona em três animais do EP, entre três e 12 horas após o término da cirurgia, enquanto nos tratamentos IM e S foram necessários nove e 11 resgates, em seis animais, respectivamente, entre uma e 12 horas após o término da cirurgia (Fig. $1)$.

Não houve diferença significativa entre os grupos em relação à concentração sérica de cortisol (Tab. 3). No tratamento $\mathrm{S}$, valores mais baixos foram observados às 12 horas em relação às seis horas após o término do procedimento cirúrgico.

Com relação aos efeitos indesejáveis, ocorreram sinais de euforia em apenas dois animais (um do EP e um do IM) durante a recuperação pósanestésica. Em 50\% e $75 \%$ dos animais foram observadas midríase e hipertermia, respectivamente, com todos os tratamentos avaliados. Vômito e sialorreia não foram observados no decorrer das avaliações.

Tabela 1. Dados demográficos das gatas submetidas à ovariossalpingoisterectomia, tratadas com metadona epidural (EP), metadona intramuscular (IM) e solução salina (S)

\begin{tabular}{lccc}
\hline & EP & IM & S \\
\hline Idade (meses) & $13 \pm 0,5$ & $12 \pm 2$ & $13,5 \pm 3$ \\
Peso (kg) & $2,8 \pm 2$ & $2,7 \pm 3$ & $2,8 \pm 2$ \\
Tempo cirúrgico (min) & $35 \pm 19$ & $39 \pm 8$ & $34 \pm 8$ \\
Tempo de extubação (min) & $14 \pm 7$ & $20 \pm 8$ & $19 \pm 4$ \\
Tempo de recuperação (min) & $50 \pm 6$ & $46 \pm 11$ & $44 \pm 11$ \\
\hline
\end{tabular}

Dados expressos em média \pm SD.

Tabela 2. Valores médios e desvio padrão dos escores de dor e de sedação em gatas submetidas à ovariossalpingoisterectomia, tratadas com metadona epidural (EP), metadona intramuscular (IM) e solução salina $(\mathrm{S})$

\begin{tabular}{lcccc}
\hline & $1 \mathrm{~h}$ & $3 \mathrm{~h}$ & $6 \mathrm{~h}$ & $12 \mathrm{~h}$ \\
\hline IVAS & & & & \\
EP & $20 \pm 7$ & $26 \pm 11$ & $26 \pm 10$ & $27 \pm 12$ \\
IM & $30 \pm 16$ & $34 \pm 12$ & $36 \pm 15$ & $35 \pm 14$ \\
S & $34 \pm 24$ & $36 \pm 15$ & $44 \pm 15$ & $37 \pm 12$ \\
\hline VAS & & & & $15 \pm 6$ \\
EP & $11 \pm 6$ & $15 \pm 9$ & $14 \pm 5$ & $18 \pm 13$ \\
IM & $14 \pm 9$ & $16 \pm 7$ & $20 \pm 11$ & $19 \pm 6$ \\
S & $21 \pm 21$ & $18 \pm 12$ & $21 \pm 10$ & \\
\hline Grau de sedação & & & & ----- \\
EP & $1 \pm 0,8$ & $0,3 \pm 0,5$ & $0,25 \pm 0,5$ & ---- \\
IM & $1,4 \pm 1$ & $0,6 \pm 0,5$ & ---- & ---- \\
S & $2 \pm 1,6$ & $0,6 \pm 0,5$ & ---- & \\
\hline
\end{tabular}

IVAS = Escala Analógica Visual Interativa; VAS= Escala Analógica Visual. 
Tabela 3. Valores médios e desvio padrão da concentração sérica de cortisol ( $\mu \mathrm{g} / \mathrm{dL})$ em gatas submetidas à ovariossalpingoisterectomia, tratadas com metadona epidural (EP), metadona intramuscular (IM) e solução salina (S)

\begin{tabular}{lccc}
\hline Tratamento & Basal & $6 \mathrm{~h}$ & $12 \mathrm{~h}$ \\
\hline EP & $1,92 \pm 1,2$ & $2,69 \pm 1,1$ & $1,39 \pm 1,4$ \\
IM & $1,85 \pm 1,2$ & $2,0 \pm 1$ & $1,04 \pm 0,47$ \\
S & $1,35 \pm 0,9$ & $2,3 \pm 1,1$ & $0,97 \pm 0,65^{*}$ \\
\hline
\end{tabular}

*Redução significativa em relação às seis horas pós-operatória $(\mathrm{P}<0,05)$.

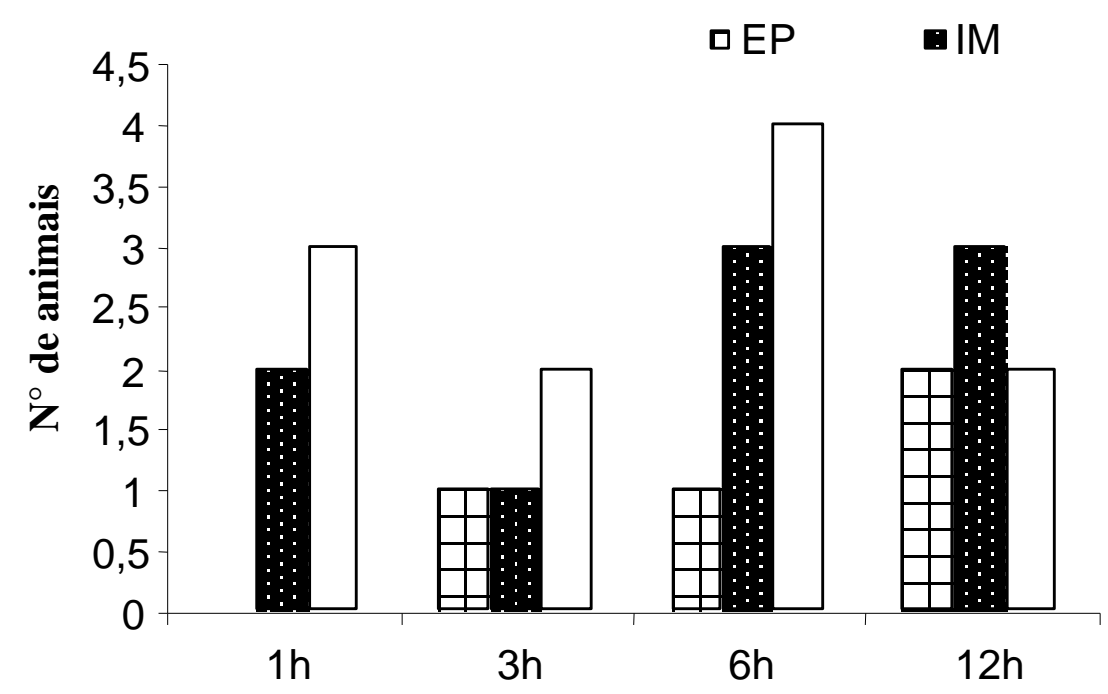

Figura 1. Número de gatos que receberam resgate de metadona ao longo da avaliação analgésica (um, três, seis e 12 horas após o término da cirurgia) nos tratamentos EP $\left(0,2 \mathrm{mg} \mathrm{kg}^{-1}\right.$ de metadona via epidural), IM ( $0,2 \mathrm{mg} \mathrm{kg}^{-1}$ de metadona via intramuscular) e $S\left(0,2 \mathrm{~mL} \mathrm{~kg}^{-1}\right.$ de solução salina, via epidural e intramuscular).

\section{DISCUSSÃO}

A dor é difícil de ser diagnosticada em gatos, em função de sinais inexpressivos de desconforto, bem como devido à ausência de outras alterações fisiológicas (Gassel et al., 2005). Estudos prévios têm demonstrado que a escala analógica visual (VAS) e a escala analógica visual interativa (IVAS) são métodos sensíveis para avaliação de dor em gatos, sobretudo quando a palpação da área operada é utilizada para a mensuração de desconforto (Roher Bley et al., 2004; Brondani et al., 2009).

No presente estudo, não houve diferenças entre os tratamentos nos escores de dor pós-operatória, concordando com os resultados relatados por Gassel et al. (2005) em pesquisa realizada em gatas para avaliação de dor após OSH. O primeiro resgate foi efetuado na terceira hora pós-operatória, período correspondente a $235 \pm 9$ minutos após a administração da metadona, semelhante ao estudo prévio realizado em cães, no qual o primeiro resgate analgésico após administração epidural desse opioide foi necessário aos 241 minutos (Leibetseder et al., 2006). Paralelamente, nos animais tratados com metadona intramuscular, em três animais o resgate foi necessário na primeira hora de avaliação pós-operatória, que correspondeu a um período médio de $115 \pm 8$ minutos da administração da metadona, sendo esse resultado semelhante ao observado por Roher Bley et al. (2004), que relataram analgesia satisfatória em período máximo de até 130 minutos, após a administração intramuscular desse opioide na dose de $0,3 \mathrm{mg} \mathrm{kg}^{-1}$ em $95 \%$ dos gatos avaliados.

Apesar de a administração epidural da metadona ter possibilitado um período de analgesia mais 
longo em relação à intramuscular, quando comparada com opioides hidrossolúveis, como a morfina, esse efeito é pouco relevante. A metadona é 82 vezes mais lipossolúvel em relação à morfina, de modo a determinar curta duração de ação (Cousins e Mather 1984), conforme demonstrado em estudo prévio desenvolvido no homem, ao verificar efeito analgésico de cinco a oito horas com o uso da metadona epidural, enquanto a morfina epidural promoveu analgesia por período de até 16 horas (Bromage et al., 1980). Em gatos, não há relatos na literatura em relação à duração do efeito analgésico da metadona pela via epidural. No entanto, a administração epidural de morfina nessa espécie culminou com aumento no limiar nociceptivo térmico até 16 horas, sugerindo efeito antinociceptivo de longa duração (Pypendop et al., 2008).

No atual estudo, $50 \%, 25 \%$ e $25 \%$ dos animais tratados com metadona epidural, metadona intramuscular e salina, respectivamente, não receberam analgesia complementar durante o período de 12 horas. Dessa forma, apesar de a OSH ser um excelente modelo para padronização da dor aguda pós-operatória em animais, é possível que este tipo de procedimento não estimule desconforto suficiente para que a dor discreta a moderada seja avaliada em gatos, visto que esses animais apresentam sutileza na forma de expressar os sinais de dor. Além desses fatores, as escalas empregadas para aferição de dor nesse estudo podem não ter sido sensíveis o bastante para detectar diferenças sutis de comportamento entre os gatos dos diferentes tratamentos, semelhante aos achados de Gassel et al. (2005).

$\mathrm{Na}$ primeira hora de avaliação pós-operatória, esperava-se que os animais tratados com solução salina expressassem sinais de desconforto, sendo necessária a analgesia de resgate, visto que nenhum fármaco com propriedade analgésica foi empregado no protocolo anestésico. No entanto, apenas três gatas $(37,5 \%)$ demonstraram desconforto suficiente para que a analgesia suplementar fosse efetuada. Todavia, é difícil distinguir a analgesia da sedação, em função do efeito sedativo residual dos fármacos empregados no protocolo anestésico, de modo que alguns animais podem não expressar sinais de dor, bem como não responder ao estímulo tátil efetuado na área de incisão cirúrgica. Dessa forma, é esperado que a sedação possa mascarar a dor nas mensurações realizadas em até uma hora após a cirurgia (Roher Bley et al., 2004).

O grau de sedação bem como o tempo de extubação e de recuperação pós-anestésica não foram alterados pela administração préoperatória da metadona, sugerindo que esse opioide induziu mínima depressão central em gatos, confirmando estudos prévios, cuja administração da metadona não determinou alterações comportamentais relevantes (Dobromylskyj, 1993; Steagall et al., 2006; Monteiro et al., 2008).

Euforia e episódios de excitação não foram observados com frequência no presente estudo, semelhante a relatos prévios (Steagall et al., 2006; Ferreira, 2010). Os opioides podem induzir sinais de excitação em gatos, sobretudo em função do uso de doses elevadas. Porém, no atual estudo, a dose empregada foi mais baixa que as doses utilizadas por outros pesquisadores (Dobromylskyj, 1993; Rohrer Bley et al., 2004; Monteiro et al., 2008), visando reduzir os possíveis efeitos de euforia e/ou excitação induzidos pela metadona (Steagall et al., 2006)

Com relação ao diâmetro pupilar, metade dos animais apresentou midríase no período pósoperatório, semelhante aos resultados de Ferreira (2010), que relatou midríase de longa duração após o uso de metadona em gatos. A midríase é um efeito bem característico dos opioides nos gatos (Gaumann et al., 1988), a qual normalmente está associada à interação desses analgésicos aos receptores $\sigma$ e, por vezes, pode ser atribuída à overdose. Ademais, é uma resposta simpática, que pode ser indicativa de dor, quando associada a outros sinais. No presente estudo, não houve relação direta entre midríase e sinais de desconforto.

A hipertermia (temperatura retal $>39,2^{\circ} \mathrm{C}$ ) observada no período pós-operatório é semelhante à relatada previamente na espécie felina, com o uso de opioides para modulação da dor (Posner et al., 2007; Brondani et al., 2009). $\mathrm{O}$ aumento da temperatura determinado pelo opioide pode ser parcialmente atribuído ao incremento das atividades muscular e locomotora, contudo um mecanismo específico hipotalâmico também tem sido identificado (Branson et al., 2001). 
Estudos prévios têm demonstrado que a administração de opioides em associação à anestesia geral favorece a redução da liberação hormonal em resposta ao estresse pósoperatório, inibindo a liberação do hormônio adrenocorticotrófico (ACTH) e secundariamente a de cortisol (Gaumann et al., 1988; Leibetseder et al., 2006). No atual estudo, o cortisol mantevese dentro dos limites fisiológicos para a espécie felina, os quais estão compreendidos entre 0,33$2,57 \mu \mathrm{g} \mathrm{dL}^{-1}$ (Kaneko et al., 1997), sendo observado comportamento semelhante no decorrer do tempo e entre os grupos, provavelmente em função dos resgates de metadona efetuados ao longo da avaliação da dor pós-operatória. Dessa forma, mesmo nos animais tratados com solução salina foi demonstrada modulação neuroendócrina, com valores fisiológicos de cortisol no período pósoperatório, sugerindo que a metadona inibiu o eixo hipotálamo-pituitária-adrenal, de modo a modular a resposta de estresse pós-cirúrgica, concordando com os achados descritos no cão (Leibetseder et al., 2006).

\section{CONCLUSÕES}

A administração de metadona pela via epidural resultou em menor requisito analgésico pósoperatório, bem como analgesia mais prolongada, quando comparada à via intramuscular. Mínimos efeitos sedativos foram identificados com o uso da metadona. Paralelamente, houve modulação da resposta de estresse, independentemente do uso pré ou pósoperatório, bem como da via de administração da metadona em gatas submetidas à $\mathrm{OSH}$.

\section{REFERÊNCIAS}

BRANSON, K.R.; GROSS, M.E.; BOOTH, N.H. Opioids agonists and antagoinists. In: ADAMS, H.R. (Ed.) Veterinary Pharmacology and Therapeutics. Ames: States Press, 2001. p.274-310.

BROMAGE, P.R.; CAMPORESI, E.; CHESTNUT, D. Epidural narcotics for postoperative analgesia. Anesth. Analg., v.59, p.473-480, 1980.

BRONDANI, J.T.; LUNA, S.P.; BEIER, S.L. et al. Analgesic efficacy of perioperative use of vedaprofen, tramadol or their combination in cats undergoing ovariohysterectomy. J. Feline Med. Surg., v.11, p.420429, 2009.
CAPNER, C.A.; LASCELLES, B.D.; WATERMANPEARSON, A.E. Current British veterinary attitudes to perioperative analgesia for cats and small mammals. Vet. Rec., v.145, p.601-604, 1999.

COUSINS, M.J.; MATHER, L.E. Intrathecal and epidural administration of opioids. Anesthesiology, v.61. p.276-310, 1984.

DOBROMYLSKYJ, P. Assessment of methadone as an anaesthetic premedicant in cats. J. Small Anim. Pract., v.34, p.604-608, 1993.

FERREIRA, T.H. A farmacocinética da metadona e seus efeitos antinociceptivos, comportamentais e sobre a concentração alveolar mínima de sevofluorano em felinos. 2010. 118f. Tese (Doutorado em Anestesiologia) - Faculdade de Medicina, Universidade Estadual Paulista, Botucatu, SP.

GASSEL, A.D.; TOBIAS, K.M.; EGGER, C.M. et al. Comparison of oral and subcutaneous administration of buprenorphine and meloxicam for preemptive analgesia in cats undergoing ovariohysterectomy. $J$. Am. Vet. Med. Assoc., v.227, p.1937-1944, 2005.

GAUMANN, D.M.; YAKSH, T.L.; TYCE, G.M. et al. Opioids preserve the adrenal medullary response evoked by severe hemorrhage: studies on adrenal catecholamine and met-enkephalin secretion in halothane anesthetized cats. Anesthesiology, v.68, p. 743-753, 1988.

GOURLAY, G.K.; WILLIS, R.J.; LAMBERTY, J. A double-blind comparison of the efficacy of methadone and morphine in postoperative pain control. Anesthesiology, v. 64, p.322-327, 1986.

HEWSON, C.J.; DOHOO, I.R.; LEMKE, K.A. Perioperative use of analgesics in dogs and cats by Canadian veterinarians in 2001. Can. Vet. J., v.47, p.352-359, 2006.

JOUBERT, K.E. Anaesthesia and analgesia for dogs and cats in South Africa undergoing sterilisation and with osteoarthritis--an update from 2000. J. S. Afr. Vet. Assoc., v.77, p.224-228, 2006.

KANEKO, J.J.; HARVEY, J.W.; BRUSS, M.L. Clinical Biochemistry of Domestic Animals. New York, Academic, 1997. 932p.

LEIBETSEDER, E.N.; MOSING, M.; JONES, R.S.A. Comparison of extradural and intravenous methadone on intraoperative isoflurane and postoperative analgesia requirements in dogs. Vet. Anaesth. Analg., v.33, p.128-136, 2006.

MANFRINATE, R.; DAHROUG, M.A.A., FARIAS, D.C. et al. Efeitos da morfina e da metadona associadas à acepromazina em gatas anestesiadas com propofol e halotano e submetidas à ovariossalpingoisterectomia. Acta Sci. Vet., v.37, p.245-251, 2009. 
MONTEIRO, E.R.; PICOLI, F.M.; OLIVEIRA, M.G. et al. Efeitos sedativo e cardiorrespiratório da administração da metadona, isoladamente ou em associação à acepromazina ou xilazina, em gatos. Braz. J. Vet. Res. Anim. Sci., v.45, p.289-297, 2008.

POSNER, L.P.; GLEED, R.D.; ERB, H.N. et al. Postanesthetic hyperthermia in cats. Vet. Anaesth. Analg., v. 34, p.40-47, 2007.

PYPENDOP, B.H.; SIAO, K.T.; PASCOE, P.J. et al. Effects of epidurally administered morphine or buprenorphine on the thermal threshold in cats. Am. J. Vet. Res., v.69, p. 983-987, 2008.

ROHRER BLEY, C.; NEIGER-AESCHBACHER, G.; BUSATO, A. et al. Comparison of perioperative racemic methadone, levo-methadone and dextromoramide in cats using indicators of postoperative pain. Vet. Anaesth. Analg., v.31, p.175-182, 2004.
STEAGALL, P.V.; CARNICELLI, P.; TAYLOR, P.M. et al. Pressure and thermal thresholds in cats: effects of methadone, buprenorphine and morfine. $J$. Vet. Pharmacol. Ther., v.29, p.531-535, 2006.

STEAGALL, P.V.; TAYLOR, P.M.; RODRIGUES, L.C., et al. Analgesia for cats after ovariohysterectomy with either buprenorphine or carprofen alone or in combination. Vet. Rec., v.164, p.359-363, 2009.

WATSON, A.D.; NICHOLSON, A.; CHURCH, D.B. et al. Use of anti-inflammatory and analgesic drugs in dogs and cats. Aust. Vet. J., v.74, p.203-210, 1996.

ZAR, J.H. Biostatistical Analysis. New Jersey: Prentice-Hall Upper Saddle River. 1996, 718p. 